\title{
Uma análise comparativa entre sistemas de inovação e o diamante de Porter na abordagem de arranjos produtivos locais
}

\author{
Sebastião Décio Coimbra de Souza \\ José Arica \\ Universidade Estadual do Norte Fluminense - UENF
}

\begin{abstract}
Resumo
Neste trabalho apresenta-se uma avaliação comparativa entre as abordagens de Sistemas de Inovação e o Diamante de Porter para análise da competitividade de aglomerados e sistemas produtivos locais. Inicialmente, é feita uma revisão das abordagens mais recentes para análise de sistemas produtivos locais, regionais ou setoriais, considerando conceitos evolucionários. A seguir, as características distintivas das abordagens de Sistemas de Inovação e do Diamante de Porter são comparadas e comentadas. Em síntese, enquanto a abordagem de Sistemas de Inovação enfatiza as características de cooperação e interação entre instituições que suportam o desenvolvimento e o avanço tecnológico e organizacional, o Diamante privilegia o contexto competitivo local/setorial e os aspectos de mercado em perspectiva internacional. Ambas as abordagens consideram a infra-estrutura e os fluxos institucionais (formais e informais) como precondição fundamental para o desenvolvimento econômico. Como uma alternativa para suprir certas lacunas verificadas em ambas as abordagens na análise da competitividade em arranjos produtivos locais, sugere-se uma perspectiva evolucionária.
\end{abstract}

Palavras-chave

Arranjos produtivos locais, sistemas de inovação, diamante de Porter, abordagem evolucionária.

\section{A comparative analysis between innovation systems and Porter's diamond on the local clusters approach}

\begin{abstract}
In this work is presented a comparative evaluation between Innovation Systems and Porter's Diamond approaches for competitive analysis in Clusters and Local Productive Systems. Initially it is done a brief review of the recent approaches to regional, local and sectorial productive systems considering evolutionary technical-economic concepts. Then, the distinctive characteristics between Innovation Systems and Porter's Diamond approaches are compared and commented. In synthesis, while the Innovation System approach the characteristics of cooperation and interaction between institutions that support the development and the technological and organizacional advance, the Diamond privileges the local/setorial competitive context and the aspects of international market. However, the infra-structure and the formal and informal institutional flows are essential for the economic development on both approaches. Finally, as an alternative to suply some gaps verifyed on both approaches on the local clusters competitive analysis, an evolutionary perspective is suggested.
\end{abstract}

Key words

Innovation systems, Porter's diamond, local productive systems, Evolutionary perspective. 


\section{INTRODUC̣̃̃O}

A intensificação da competição internacional tem forçado as empresas a uma rápida absorção de novas tecnologias para manter ou ampliar mercados e se manterem competitivas. No contexto da competição internacional, o desempenho econômico nacional ou regional depende fundamentalmente do grau de utilização das bases existentes de tecnologia, de treinamento profissional e científico, e da exploração econômica eficiente dessas bases. A competitividade das firmas é cada vez mais determinada pela tecnologia dominada e pela capacidade de adaptação a mudanças (DOSI, 1988; BAUM \& SINGH, 1994).

Segundo Porter (1989), o desenvolvimento de processos e produtos tecnológicos mais avançados, eventualmente inovadores, é um dos principais pilares para uma empresa obter vantagens competitivas no atual cenário econômico, respondendo às contínuas mudanças exigidas pelo mercado.

Nesse sentido, os conceitos tradicionais de algumas variáveis envolvidas no contexto da competitividade têm sido revistos. A "tecnologia", antes vista como simples elemento residual, tem agora seu conceito ampliado, passando a envolver múltiplas facetas. $\mathrm{O}$ avanço tecnológico tem movido o conceito padrão de choques exógenos para um crescente entendimento dos elementos endógenos na criação de novas tecnologias e estímulos ao crescimento (STORPER, 1997; LUNDVALL, 1992; CHANDLER Jr. et al., 1999).

A visão tradicional de inovação tecnológica linear e unidimensional baseada nos conceitos de science push e technology pull ou marketing pull, tem sido ampliada, e, em alguns casos, substituída por uma apreciação mais complexa, incorporando diferentes aspectos além daqueles tradicionais de alta e baixa tecnologia e de tecnologia do produto e de processo (NORTH \& SMALLBONE, 2000). Neste aspecto, tecnologia e inovação são vistos mais como um processo interativo entre homem, organização e contexto ambiental, e relacionados a ativos invisíveis e a conceitos evolucionários (DOSI, 1982; 1988; EDQUIST, 1997; ZIMAN, 2000; SAVIOTTI \& NOOTEBOOM, 2000). Assim, conceitualmente, o termo "inovação tecnológica" muda de sentido, pois a "inovação" descola-se do conceito tradicional de "tecnologia" para uma visão mais ampla e sistêmica, incorporando aspectos ambientais, políticos, sociais e culturais, além dos de mercado.

Paralelamente, a infra-estrutura e o ambiente regional/local assumem papel determinante nas novas aborda- gens do desenvolvimento econômico. Muitos estudos nesse sentido têm tido destaque na literatura (ver, por exemplo, PORTER, 1989; MALECKI, 1997; MALECKI \& OINAS, 1998; PACI \& USAI, 2000). Entretanto, estes estudos focam quase que apenas em países centrais e exclusivamente em setores industriais altamente avançados, que poderiam ser posicionados no nível mais elevado de uma escala de evolução tecnológica hipotética, cujo fator determinante da competitividade é a inovação. O emprego dessas abordagens e metodologias, ipsis litteris, ao estudo de arranjos produtivos locais (aglomerados de forma geral) de indústrias tradicionais, menos densos em termos de tecnologia, tem gerado um vazio na interpretação e pouco contribuído para o entendimento da dinâmica do desenvolvimento de sistemas produtivos locais de regiões periféricas. Há a necessidade de criação de abordagens que captem as diversidades locais e regionais e as diferenças entre firmas de um mesmo arranjo produtivo em termos de dinâmica competitiva e tecnológica.

\section{A abordagem de SI enfatiza os aspectos A institucionais que formam um ambiente fértil e as interações entre os atores que propiciam o surgimento de inovações.}

A partir de uma revisão das abordagens mais difundidas na literatura para o estudo e análise da dinâmica do desenvolvimento de certas aglomerações produtivas e clusters locais, comentam-se na seção seguinte, em mais detalhes, as abordagens de Sistemas de Inovação e o Diamante de Porter, com ênfase nas características distintivas entre ambas. Mais adiante, ressalta-se a preeminência de uma integração dos conceitos dessas duas abordagens através de um conjunto de hipóteses gerais que caracterizam uma abordagem evolucionária. Em seguida, sugere-se o tratamento de arranjos produtivos locais através de uma perspectiva baseada em princípios evolucionários. Por fim, são apresentadas as considerações finais.

\section{A ANÁLISE DE SISTEMAS INDUSTRIAIS E A ABORDAGEM EVOLUCIONÁRIA}

Para se estudar, entender e caracterizar a dinâmica da infra-estrutura envolvida no processo de avanço tecnológico (científica, tecnológica, econômica e social), têm sido propostos novos conceitos e abordagens metodoló- 
gicas, principalmente na dimensão nacional (PORTER, 1989; LUNDVALL, 1992; NELSON, 1993). Mais recentemente, vários autores têm tentado estender estes conceitos para análise de regiões (subnacionais) e setores (de la MOTHE \& PAQUET, 1998). Uma característica comum a todas estas abordagens é que a "força-motriz" do desenvolvimento econômico está nas inovações, que pressupõe um sistema tecnológico, como pioneiramente defendido por Schumpeter (1934) e, posteriormente, ampliado pelos neo-schumpeterianos.

série de estudos utilizando o conceito de "Sistema Nacional de Inovação" (LUNDVALL, 1992; NELSON, 1993). Nestes trabalhos, há uma ênfase nas interações entre instituições e atores, principalmente aqueles ligados à área de Ciência e Tecnologia (C\&T). A análise foca no papel da política tecnológica em diferentes países, considerando um certo período de tempo. Estas análises envolvem instituições de Pesquisa e Desenvolvimento (P\&D), Universidades, Institutos de Pesquisa, agências e políticas de governo. A firma é apresentada como um núcleo de habilidades, competências e conhecimentos tecnológicos e organizacionais (FREEMAN, 1994).

A partir desta abordagem, os autores desta corrente identificaram, e passaram a considerar, formas diferentes de aprendizado (learning by doing, by using, by interacting, by searching, entre

Schumpeter (1934), em seus estudos seminais sobre o papel do avanço tecnológico sobre a economia, distinguiu cinco tipos de inovações: (i) introdução de um novo produto ou uma modificação qualitativa em um já existente; (ii) novo processo para uma indústria; (iii) abertura de um novo mercado; (iv) desenvolvimento de novas fontes de suprimento de matéria-prima ou outros insumos; e, (v) mudanças em uma organização industrial. Na análise evolucionária ou neo-schumpeteriana, o processo de inovação das firmas é determinado por seu comportamento, através de sua capacidade de absorver conhecimento, aprender a partir de experiências exógenas e endógenas, individuais e coletivas, de concepção, produção e comercialização de bens e serviços e de interações com outras organizações e instituições (FREEMAN, 1994; METCALFE, 1995; DOSI \& NELSON, 1996; SAVIOTTI \& NOOTEBOOM, 2000).

Erik Dahmém, ao incorporar uma visão de sistema em seu estudo clássico sobre as dinâmicas das concentrações urbanas industriais na Suécia (DAHMÉN, 1950), as quais denominou de "Blocos de Desenvolvimento", provavelmente realizou o primeiro trabalho com uma abordagem baseada em conceitos da dinâmica evolucionária schumpeteriana. Dahmém focou no papel do empreendedorismo e dos empreendedores, e nas condições requeridas para se poder tirar vantagem de novas oportunidades de negócios, o que provocaria tensões entre recursos disponíveis e a capacidade de explorá-los comercialmente. Depois desse estudo pioneiro, houve um longo período até surgirem novas abordagens de sistemas na literatura econômica industrial (CARLSSOM, 1997).

Porém, ao longo da década de 90, foi publicada uma outras). Posteriormente, foi observado também que a taxa de aprendizado depende fundamentalmente de um conjunto de interações com múltiplas fontes internas e externas de informação e conhecimento. Essas interações criariam uma interdependência sistêmica, não formal, nos sistemas produtivos dos respectivos atores, dando origem ao que passou a ser denominado de "Sistema de Inovação" (ver, por exemplo, LUNDVALL, 1992; EDQUIST, 1997). Conseqüentemente, a estrutura produtiva do ambiente considerado (país ou região), com seus aspectos históricos e culturais, condicionaria tal sistema (COOKE \& MORGAN, 1998).

Outra abordagem amplamente conhecida, e talvez a mais empregada para análise de aglomerados produtivos, é o chamado "Diamante de Porter", descrita no livro de Michael Porter A vantagem competitiva das nações (Porter, 1989), a partir de estudos empíricos em diversos países. Esta foca essencialmente em clusters (aglomerados industriais altamente dinâmicos), suas estratégias e competitividade. Os quatro lados do Diamante são constituídos por: (i) condições de fatores (recursos, habilidades, tecnologias, capital, etc.), (ii) condições de demanda, (iii) elos para relacionar e auxiliar indústrias e, (iv) estratégia das firmas, estrutura e competição. Cada atividade econômica é vista como parte de um aglomerado de atividades e agentes, ao invés de isoladamente.

Saxenian (1994) utiliza uma abordagem em torno do conceito denominado de "Sistemas de Inovação Local". Através de um estudo da indústria eletrônica do Vale do Silício, Califórnia, e ao longo da Route 128, em Massachussets, seu trabalho focou nas diferenças de cultura e competição, grau de hierarquia e concentração, 
experimentação, colaboração e no aprendizado coletivo, o que teria provocado diferenças na capacidade de ajuste a mudanças nas circunstâncias de mercado naqueles dois pólos tecnológicos americanos. Contudo, esse trabalho não propôs nem adotou uma abordagem própria para o estudo desses sistemas, limitando-se apenas ao estudo e descrição das diferenças dos processos de surgimento e de crescimento dos aglomerados estudados.

Outra proposta para análise da competitividade de aglomerados setoriais é denominada de "Sistemas Setoriais de Inovação" (BRESCHI \& MALERBA, 1997; MALERBA, 2001). Este conceito baseia-se na idéia de que diferentes setores (ou indústrias) operam sob certos regimes tecnológicos que são caracterizados por combinações particulares de oportunidades e condições de apropriabilidade, graus de cumulatividade de conhecimento tecnológico e fatores relativos às características específicas do conhecimento requerido em certos setores econômicos. Apesar da denominação, esta proposta é mais próxima, e até similar em alguns pontos, ao modelo de Porter, focando nas relações competitivas entre firmas e procurando considerar explicitamente o papel da seleção pelo ambiente.

Vale destacar que, no Brasil, uma série de estudos tem sido desenvolvida sob a denominação de "Arranjos e Sistemas Produtivos Locais", utilizando o conceito geral de Sistemas de Inovação, aplicado a aglomerados locais (CASSIOLATO \& LASTRES, 2000). Estes trabalhos dão destaque para o levantamento histórico de formação desses arranjos, à análise da influência das instituições de suporte às atividades de ciência e tecnologia, e à proposição de políticas públicas de apoio e sustentação desses sistemas.

Neste caso, os principais fatores considerados são: (i) a dimensão local do aprendizado, da capacitação e da inovação; (ii) o processo de globalização e os sistemas nacionais, supra e subnacionais de inovação; (iii) o papel de arranjos produtivos locais e sua capacidade; e (iv) o novo papel e objetivos das políticas de desenvolvimento científico e tecnológico, tendo em vista as dimensões supranacional, nacional, regional, estadual e local. Encontram-se também incluídos nesta série outros trabalhos que utilizam a abordagem de cadeias produtivas e redes (ver, por exemplo, BERNARDES, 2000).

Entretanto, nesses estudos não é oferecida uma proposta conceitual adaptada às questões locais e a análise limita-se à esfera empírica. Na maior parte dos casos, a configuração e a própria existência de um "Sistema de Inovação Local" não fica clara.
Em um artigo publicado em meados da década de 90, Meyer-Stamer (1995) já questionava a existência de um "Sistema" de Inovação Industrial no Brasil. Sua argumentação baseia-se no fato de haver pouca interação entre as universidades e centros de pesquisa e o meio empresarial, o que, segundo o autor, justificaria o baixo desempenho do país em termos de inovações e patentes. Nesse sentido, o trabalho de Maculan \& Carleial (1999) também chegou a conclusões semelhantes, através do estudo do processo de aprendizagem em redes de subcontratação de micro e pequenas empresas e incubadoras no sul do país.

A seguir, as abordagens de Sistemas de Inovação e o Diamante de Porter, são consideradas em mais detalhes.

\section{SISTEMAS DE INOVAC̣̃̃O E O DIAMANTE DE PORTER}

A abordagem de Sistemas de Inovação (SI) não assume como hipótese básica uma relação de causa e efeito, determinística, nem pretende prover respostas, por si mesma, de como o sistema evolui no tempo. Conseqüentemente, não indica ferramentas ou métodos a empreender na análise dos sistemas. O conceito de SI é visto como "holístico", e, como tal, em termos metodológicos surgem dificuldades analíticas para sua aplicação.

\section{A mbas as abordagens (SI e o Diamante) - compartilham similaridades, como na tendência em focar os fundamentos para entender o comportamento dos sistemas.}

De uma forma geral, a abordagem de SI enfatiza os aspectos institucionais e histórico-culturais que formam um ambiente fértil em idéias e as interações entre atores público-privados, que propiciam o surgimento de inovações. Nesta abordagem há um predomínio absoluto de pesquisas empíricas que buscam identificar e diagnosticar fatores e características que proporcionaram o surgimento do denominado "sistema de inovação", como o Vale do Silício (SAXENIAN, 1994), País Basco, Emilia Romagna, Baden-Württemberg (COOKE \& MORGAN, 1998), além de outros casos de regiões prósperas a partir de aglomerados industriais.

Entretanto, como destacado por Radosevic (1998), permanecem ainda algumas questões problemáticas na conceituação de SI, como por exemplo: (i) a definição dos limites do sistema; e (ii) o tratamento da diversidade institucional. 
De modo a caracterizar melhor as similaridades e as diferenças entre a abordagem de SI e as mais tradicionais, utilizaremos como referencial o modelo "Diamante" de Porter (1989), amplamente difundido e empregado na análise de competitividade de setores industriais em diversos países (para o caso do Brasil ver, por exemplo, COUTINHO \& FERRAZ, 1994).

Em sua abordagem, Porter (PORTER, 1989: xvi) busca uma nova visão da vantagem competitiva centrada na habilidade de uma nação em utilizar produtivamente seus insumos e recursos através da formação de clusters. Pelo desenvolvimento do conhecimento como uma vantagem competitiva dinâmica, que para o autor pode ser criada, a análise é efetuada em vários níveis, através de elementos da economia de uma nação, focalizando clusters industriais e as interações entre os fatores do "Diamante". Dessa forma, é presumido que para uma economia nacional, o estado do sistema (ou seja, seu desempenho comercial e sua prosperidade) pode ser razoavelmente explicado pela análise dos estados dos seus elementos e suas relações complexas. Entretanto, ficam evidentes outras preocupações: “(...) meramente usar os recursos disponíveis, ou reunir mais recursos não é suficiente para alcançar prosperidade. Nem redistribuir as riquezas da nação entre grupos de interesse" (PORTER, 1989: xii). Há uma forte atenção na busca dos micro-fundamentos dos fenômenos macroeconômicos do desenvolvimento: “(...) Reformas macroeconômicas são necessárias, mas não suficientes (...)". Tão importantes são os alicerces microeconômicos que constituem o ambiente onde as empresas competem, de modo a responder às seguintes questões: Qual o próximo passo? O que fazer após o ajuste macroeconômico e a estabilização..." (PORTER, 1989: xvi).

Como aspectos disciplinares distintivos entre as duas abordagens tratadas, podemos citar que, enquanto a abordagem de SI está mais vinculada ao processo de inovação tecnológica (IT) e ao campo da C\&T, o Diamante de Porter, refere-se essencialmente à arena competitiva comercial e à gestão empresarial. Como métodos analíticos, ambas as abordagens compartilham similaridades, como na tendência em focar os fundamentos para entender o comportamento dos sistemas.

Por outro lado, há uma dificuldade de se trabalhar estas abordagens em sistemas produtivos tecnologicamente defasados, nos quais não se pode considerar um "sistema de inovação", ou em que os fatores do "Diamante" não são plenamente estabelecidos. Nestes ambientes, fatores mais "primitivos", mas importantes para a formação da competitividade das empresas e o fortalecimento dos aglomerados, são condições essenciais para o crescimento econômico do sistema, e não podem ser negligenciados. Entre tais fatores, podem ser incluídos aspectos operacionais (como a redução dos desperdícios, o aumento da produtividade e da qualidade dos produtos e serviços, além de outros), além de alguns estruturais (como a formação de coalizões para a eliminação de barreiras à absorção de novas tecnologias e a cooperação institucional).

O Quadro 1, a seguir, sintetiza as principais características analíticas entre as abordagens de Sistemas de Inovação e o modelo "Diamante".

Quadro 1: Aspectos característicos distintivos entre SI e o modelo de Porter.

\begin{tabular}{|c|c|c|}
\hline CRITÉERIO & SISTEMAS DE INOVAÇÃO & DIAMANTE DE PORTER \\
\hline Hipótese central & $\begin{array}{l}\text { A melhoria na eficiência da aprendizagem é a } \\
\text { fonte para a "inovatividade" de uma nação. }\end{array}$ & $\begin{array}{l}\text { O crescimento da produtividade é a } \\
\text { fonte da prosperidade de uma nação. }\end{array}$ \\
\hline Unidade analítica & $\begin{array}{l}\text { Nação, região, setor; aglomerado (indústria, } \\
\text { região]; Firma; Instituições de conhecimento } \\
\text { público (infra-estrutura educacional e de C\&T); } \\
\text { Redes de transferência de conhecimento; } \\
\text { Sistema tecnológico. }\end{array}$ & $\begin{array}{l}\text { Nação; Cluster [indústria, região]; } \\
\text { Firmas em setores industriais. }\end{array}$ \\
\hline $\begin{array}{l}\text { Processo } \\
\text { dinâmico/causa }\end{array}$ & $\begin{array}{l}\text { Entre tecnologia e instituição: Trajetórias } \\
\text { tecnológicas; Teoria do ciclo de vida da } \\
\text { tecnologia; Dependência da trajetória (path- } \\
\text { dependence); Learning by doing, by using, and by } \\
\text { interaction; Transferência de conhecimento. }\end{array}$ & $\begin{array}{l}\text { Entre os elementos do diamante: } \\
\text { Estratégias das firmas; Estrutura e } \\
\text { concorrência; Condições de fatores; } \\
\text { Indústrias vinculadas e auxiliares. }\end{array}$ \\
\hline Disciplina vinculada & Inovação tecnológica & Gestão empresarial \\
\hline Direcionamento & C\&T & Comércio e negócios \\
\hline
\end{tabular}

Fonte: Souza \& Arica (2003b) 


\section{PARA UMA ABORDAGEM EVOLUCIONÁRIA DE ARRANJOS PRODUTIVOS LOCAIS}

Avanços no estudo do desenvolvimento econômico local e das novas organizações produtivas industriais têm mostrado que há a necessidade de se estabelecer uma nova fronteira de pesquisa nesse campo. Neste caso, a visão tradicional de divisão dos fenômenos econômicos em microeconomia e macroeconomia precisa ser complementada pela perspectiva de uma outra dimensão (mesoeconômica), vinculada especificamente aos aspectos de localização, aglomeração e organização industrial e de vantagens competitivas e desempenho empresarial. Esta dimensão interage e é condicionada pelas dimensões micro e macro do ambiente econômico, social e ambiental, mas que, todavia, não é captada pelas abordagens tradicionais.

Por trás de ambas as abordagens discutidas anteriormente estão implícitos ou explícitos conceitos dinâmicos e evolucionários. No caso dos Sistemas de Inovação o conceito de dependência da trajetória, que considera a irreversibilidade do tempo, porém dentro de um contexto de diversidade tecnológica; e no caso do Diamante, a consideração de estratégias competitivas e cooperativas, que refletem uma forma de adaptação ao meio para garantir a sobrevivência.

Nesse sentido, a consideração de elementos e fatores das abordagens apresentadas através de princípios evolucionários pode contribuir para elaboração de abordagens específicas para o estudo e análise da dinâmica competitiva em arranjos produtivos locais. Conseqüentemente, algumas hipóteses de um conjunto geral que caracteriza o conceito "evolucionário" devem ser contempladas. No Quadro 2 são apresentadas tais hipóteses.

Por exemplo, uma abordagem na perspectiva evolucionária adotada em uma pesquisa realizada com o propósito de se analisar o impacto da inserção da tecno- logia do gás natural no pólo de cerâmica vermelha de Campos-RJ (SOUZA \& ARICA, 2003a), propõe uma perspectiva de abordagem da dinâmica do processo competitivo em arranjos produtivos locais pela lente evolucionária. Em tal abordagem, assume-se que oportunidades e mudanças tecnológicas proporcionadas pelo ambiente local provocam um processo de estratificação competitiva entre as empresas concorrentes de um mesmo aglomerado (hipótese derivada de $\mathrm{H}_{3}$ ). No caso, as empresas do arranjo foram estratificadas segundo níveis de recursos e graus de evolução tecnológica e desempenho. Índices específicos para medir tais níveis foram modelados e levantados para uma amostra representativa da diversidade no arranjo Através de uma análise do tipo Pareto, as empresas do arranjo foram classificadas em três grupos distintos: empresas líderes (A), intermediárias (B) e retardatárias (C). Com base nos princípios evolucionários contidos nas hipóteses apresentadas, através de auditorias de manufatura nas empresas do arranjo, foram identificadas as características principais de cada grupo em relação a produtos, processo produtivo e mercado (SOUZA, 2003; SOUZA \& ARICA, 2003a).

\section{CONSIDERACÕ̃ES FINAIS}

O mapeamento da estratificação em aglomerados industriais é importante tanto do ponto de vista da organização industrial e das estratégias competitivas empresariais, como para a priorização e direcionamento de ações, programas e políticas industriais de desenvolvimento econômico-social e ambiental. Indústrias, setores, aglomerados e arranjos produtivos locais de forma geral compreendem empresas com graus diferenciados de evolução tecnológica e organizacional. Entretanto, as abordagens tradicionais para a análise desses sistemas não captam tais diversidades.

\section{Quadro 2: Hipóteses gerais propostas para análise da dinâmica competitiva em arranjos produtivos locais.}

\begin{tabular}{|l|l|}
\hline HIPÓTESE & \multicolumn{1}{c|}{ DESCRIÇÃ̃o } \\
\hline $\mathrm{H}_{1}$ & A dependência da trajetória influencia a capacidade de absorção de novas tecnologias; \\
\hline $\mathrm{H}_{2}$ & A competição é condicionada por mecanismos de seleção; \\
\hline $\mathrm{H}_{3}$ & O domínio e utilização das tecnologias disponíveis provocam diversidade entre os competidores; \\
\hline $\mathrm{H}_{4}$ & $\begin{array}{l}\text { A ocorrência de contribuições próprias (adaptaçães, alterações) afeta o desempenho (esta é uma } \\
\text { característica mais próxima ao conceito lamarckiano de evolução que ao darwiniano); }\end{array}$ \\
\hline $\mathrm{H}_{5}$ & $\begin{array}{l}\text { Na dinâmica do processo de adaptação e mudança, verifica-se uma característica de auto- } \\
\text { organização do sistema, provocada, principalmente, pela competição pela sobrevivência. Esta } \\
\text { hipótese representa uma certa "racionalidade" dos agentes (diferentemente do ambiente biológico). }\end{array}$ \\
\hline
\end{tabular}


Nesse sentido, a conjugação dos preceitos assumidos no modelo Diamante e na estrutura de Sistemas de Inovação, através de uma perspectiva evolucionária, pode prover contribuições importantes para entendimento da mudança tecnológica e da dinâmica competitiva de certos arranjos produtivos locais, preenchendo lacunas referentes à sustentação teórica.

O entendimento e a análise de fatores externos, de mercado, que condicionam o comportamento das empresas em determinados ambientes avançou bastante na literatura recente. Entretanto, ainda permanece aberta a questão de qual a relação entre os fatores internos que emulam a capacidade e competências de uma organização e os efeitos de suas estratégias e comportamento em determinados aglomerados de empresas. Neste caso, respostas para tais questões dependem de pesquisa empírica e, sobretudo, de abordagens e metodologias robustas que reflitam a diversidade de comportamentos e seus efeitos no desempenho competitivo destes sistemas.

A abordagem da estratificação de grupos de empresas conforme seus padrões de comportamento competitivo, de certa forma, resgata a idéia porteriana de "agrupamentos estratégicos", porém do ponto de vista local e dinâmico, considerando que o processo de aprendizagem e os mecanismos de seleção (tecnologias, padrões técnicos e operacionais, entre outros) condicionam o processo de estratificação em arranjos produtivos locais.

Considerando as hipóteses evolucionárias propostas, sugere-se uma nova perspectiva para se abordar tal problema. Se as especificidades internas em relação à sua cadeia de valor, produtividade, qualidade, rotinas, e outros parâmetros de formação da competitividade, seguem certos padrões e formam agrupamentos característicos, poder-se-á propor programas específicos e soluções diferenciadas para as empresas de cada grupo identificado, de acordo com suas características e necessidades, seu nível de recursos e estágio tecnológico, e suas dificuldades, considerando suas competências.

Por outro lado, devido à escassez de informações e dados desagregados, deve-se levar em conta, entretanto, a dificuldade de se trabalhar com segmentos de cadeias e aglomerados de pouca densidade, principalmente com aqueles mais defasados em termos organizacionais e tecnológicos. Para um levantamento inicial, diagnósticos do perfil técnico, econômico, organizacional e mercadológico devem ser considerados, e sempre que possível, complementados pelo levantamento de informações diretas de dados primários.

Por fim, pode-se afirmar que contribuições e avanços nessa linha de pesquisa constituem uma nova e intrigante agenda de pesquisa para o entendimento da dinâmica competitiva dos arranjos produtivos locais.

\section{Artigo recebido em 08/04/2005 \\ Aprovado para publicação em 06/10/2005}

\section{- Referências Bibliográficas}

BAUM, A. C. \& SINGH, J. V. Evolutionary dynamics of organizations. Oxford: Oxford Univ. Press, 1994.

BERNARDES, R. Redes de inovação e cadeias produtivas globais: impactos da estratégia de competição da EMBRAER no arranjo aeronáutico da região de São José dos Campos. Nota Técnica 2.3 Instituto de Economia da Universidade Federal do Rio de Janeiro - IE UFRJ, 2000.

BRESCHI, B. \& MALERBA, F. Sectoral innovation systems: technological regimes schumpeteriam dynamics and spatial boundaries. In EDQUIST C. (ed.), 1997.

CARLSSON, B. (Ed.) Technological systems and industrial dynamics. Dordrecht: Kluwer Acad. Publ., 1997.
CASSIOLATO, J. \& LASTRES, H. Local systems of innovation in Mercosur countries. Industry \& Innovation, vol. 7, n. 1, 2000, p. 33-53.

CHANDLER, Jr. A.D., HAGSTRÖM, P. \& SÖLVELL, Ö. The dynamic firm. Oxford: Oxford University Press, 1999.

COOKE, P. \& MORGAN, K. The associational economy: firms, regions and innovation. Oxford: Oxford Univ. Press, 1998.

COUTINHO, L. \& FERRAZ, J. Estudo da competitividade da indústria brasileira, Campinas: Papirus, Unicamp, 1994.

DAHMÉN, E. Swedish Industriel entrepreneurial activity, (Trad. do orig. em Alemão). Stochholm: IUI, 1950. de la MOTHE, J. \& PAQUET, G. Local and regional systems of innovation. Dordrecht: Kluwer Acad. Publ., 1998.

DOSI, G. Technological paradigms and technological trajectories, Research. Policy, v. 11, 1982, p. 147-162.

Sources, procedures and microeconomics effects of innovation. Journal of Economic Literature, v. 26, 1988, p. 1120-71.

\& NELSON, R. An introduction to evolutionary theories in economics. Journal of Evolutionary Economics, v. 4, n. 2, 1996, p. 153172.

EDQUIST, C. (ed.) Systems of innovation, technologies, institutions and organizations. London: Pinter, 1997.
FREEMAN, C. The economics of technical change. Cambridge Journal of Economics, v. 18, 1994, p. 463514.

LUNDVALL, B. (ed.) National systems of innovation: towards a theory of innovation and interactive learning, London: Pinter, 1992.

MACULAN, A. M. \& CARLEIAL, L. Pequenas empresas, aprendizaxe e sistemas nacional e rexional de innovación. Revista Galega de Economía, v. 8, n.1, 1999, p. 119 140.

MALECKI, E. J. Technology and economic development: the dynamics of local, regional and national competitiveness, Essex (UK): Addison Wesley Longman Lmtd., 1997. 


\section{- Referências Bibliográficas}

. \& OINAS, P. (eds.) Making connections: technological learning and regional economic change. Aldershot (England): Ashgate, 1998.

MALERBA, F. Sectoral systems of innovation and production: concepts, analytical framework and empirical evidence. Conference "The Future of Innovation Studies", Eindhoven University of Technology, The Netherlands, 20-23 September, 2001.

METCALFE, S. Technology systems and technology policy in a evolutionary framework. Cambridge Journal of Economics, v. 19, 1995, p. 25-46.

MEYER-STAMER, J. New departures for technology policy in Brazil. Science \& Public Policy, v. 22, n. 5, 1995 , p. $295-304$

NELSON, R. R. National innovation systems: a comparative analysis. Oxford: Oxford Univ. Press, 1993.
\& WINTER, S. An evolutionary theory of economic change. Cambridge: Harvard Univ. Press, 1982.

NORTH, D. \& SMALLBONE, D. The innovativeness and growth of rural SME's during the 1990's. Regional Science, v. 34 , n. 2,2000 , p. 145 157.

PACI, R. \& USAI, S. Technological enclaves and industrial districts: an analysis of the regional distribution of innovative activity in Europe. Regional Studies, v. 34, n. 2, 2000, p. $97-$ 114.

PORTER, M.E. A vantagem competitiva das nações. 7. ed. Rio de Janeiro: Campus, 1989.

RADOSEVIC, S. Defining systems of innovation: a methodological discussion. Technology Society, v. 20, 1998, p. $75-86$.
SAVIOTTI, P. P. \& NOOTEBOOM, B. (eds.) Technology and knowledge: from the firms to innovation systems. Cheltenham, UK: Edward Elgar, 2000.

SAXENIAN, A. Regional advantage: culture and competition in Silicon Valley and Route 128. Cambridge: Harvard Univ. Press, 1994.

SCHUMPETER, J. The theory of economic development, Trans. Redvers. Opie. (orig. German language edn., 1911), Cambridge: Harvard Univ. Press, 1934

SOUZA, S.D.C. Uma abordagem evolucionária da dinâmica competitiva em arranjos produtivos locais. Tese de Doutorado (Ciências de Engenharia, ênfase em Engenharia de Produção), UENF, Campos-RJ, 2003.
. \& ARICA, J. Análise da trajetória competitiva de empresas em um arranjo produtivo local. Em: Anais do XXIII Encontro Nacional de Engenharia de Produção (ENEGEP), UFOP, Ouro Preto-MG, 22 - 24, outubro, 2003a.

. Uma comparação entre as abordagens de Sistemas de Inovação e o Diamante de Porter para a análise de arranjos produtivos locais. Em: Anais do XXIII Encontro Nacional de Engenharia de Produção (ENEGEP), UFOP, Ouro Preto-MG, 22 - 24, outubro, 2003b.

STORPER, M. The regional world: territorial development in a global economy. NY: Guilford Press, 1997.

ZIMAN, J. (ed.) Technological innovation as an evolutionary process. Cambridge: Cambridge Univ. Press, 2000.

\section{Agradecimentos}

Os autores agradecem aos revisores pelas sugestões que contribuíram para o enriquecimento do texto.

\section{- Sobre os autores}

\section{Sebastião Décio Coimbra de Souza}

Universidade Estadual do Norte Fluminense - UENF, Laboratório de Engenharia de Produção

Endereço: Av. Alberto Lamego 2000 - CEP 28013-602

Telefone: (22) 2726-1632 ramal 224, Fax ramal 211

Email:decio@uenf.br

\section{José Arica}

Universidade Estadual do Norte Fluminense - UENF, Laboratório de Engenharia de Produção

Endereço: Av. Alberto Lamego 2000 - CEP 28013-602

Telefone: (22) 2726-1632 ramal 210, Fax ramal 211

Email: arica@uenf.br 